\title{
Dynamical Properties of Discrete-Time Background Neural Networks with Uniform Firing Rate
}

\author{
Min Wan, ${ }^{1}$ Jianping Gou, ${ }^{2}$ Desong Wang, ${ }^{1}$ and Xiaoming Wang ${ }^{1}$ \\ ${ }^{1}$ School of Mathematics and Computer Engineering, Xihua University, Chengdu 610039, China \\ ${ }^{2}$ School of Computer Science and Telecommunication Engineering, Jiangsu University, Zhenjiang 212013, China \\ Correspondence should be addressed to Min Wan; wan_min@126.com
}

Received 29 October 2012; Revised 12 March 2013; Accepted 19 March 2013

Academic Editor: Teh-Lu Liao

Copyright (C) 2013 Min Wan et al. This is an open access article distributed under the Creative Commons Attribution License, which permits unrestricted use, distribution, and reproduction in any medium, provided the original work is properly cited.

\begin{abstract}
The dynamics of a discrete-time background network with uniform firing rate and background input is investigated. The conditions for stability are firstly derived. An invariant set is then obtained so that the nondivergence of the network can be guaranteed. In the invariant set, it is proved that all trajectories of the network starting from any nonnegative value will converge to a fixed point under some conditions. In addition, bifurcation and chaos are discussed. It is shown that the network can engender bifurcation and chaos with the increase of background input. The computations of Lyapunov exponents confirm the chaotic behaviors.
\end{abstract}

\section{Introduction}

A bright red light may trigger a sudden motor action in a driver crossing an intersection: stepping on the brakes at once. The same red light may be entirely inconsequential if it appears inside a movie theater [1]. Clearly, the context information determines whether a particular stimulus will trigger a motor response. To study the neural correlate of this class of phenomena, the background neural networks were proposed in [1]. Simulations showed that the proposed networks can indeed explain the working of this phenomenon, and then it is very important to study the dynamical properties of the background networks.

Convergence is one of the most important dynamical properties of neural networks [2-5]. However, the convergence analysis for the background networks is not easy. In [1], local stability is discussed. When all neurons are firing at equal rates and the total synaptic input to all neurons is the same, the convergence of the background network is studied in [6]. In this paper, we apply the forward Euler scheme to discretize the background network with uniform firing rate and study the dynamical properties of the discretetime version. To obtain the conditions for stability and convergence, we present two lemmas and three theorems. Since the background networks proposed in [1] originate from the study of the activities of human brain and chaos is essential to normal brain functioning at many levels of activity [7-10], it is very significant to study the bifurcation and chaos of the networks. However, chaotic behavior in background networks has not been reported so far. This paper will explore the chaotic behavior of the discrete-time background network with uniform firing rate. Bifurcation diagrams and Lyapunov exponents are presented to illustrate the existence of chaotic behavior.

The rest of this paper is organized as follows. In Section 2, preliminaries will be presented. The conditions for nondivergence of the discrete-time background network will be obtained in Section 3. In Section 4, we will study the stability and convergence of the network. The bifurcation and chaos will be discussed in Section 5. Finally, conclusions are drawn in Section 6.

\section{Preliminaries}

Consider the following background network presented in [1] in which the firing rates for all neurons are equal:

$$
\tau \frac{d x}{d t}=-x+\frac{(w x+h)^{2}}{s+v N x^{2}}
$$


where $x$ is the uniform firing rate of all neurons, $\tau>0$ is a time constant, $s>0$ is a saturation constant, $v>0$ is the strength of a synaptic connection, $N$ is the total number of neurons, $h \geq 0$ represents the background input whose value is independent of the network's activity, and the total synaptic input $w>0$ to each neuron is equal.

Applying the forward Euler scheme to the network (1), we obtain the discrete-time system as follows:

$$
\tau\left[\frac{x(k+1)-x(k)}{T}\right]=-x(k)+\frac{(w x(k)+h)^{2}}{s+v N x^{2}(k)}
$$

for $k \geq 0$, where $T$ is the sampling time. That is,

$$
x(k+1)=(1-\delta) x(k)+\delta \frac{(w x(k)+h)^{2}}{s+v N x^{2}(k)}
$$

for $k \geq 0$, where $\delta=T / \tau>0$ is called step size. The following lemmas are useful.

Lemma 1. Suppose that $0<\delta<1$. The trajectories of (3) will remain in the positive regime if $x(0) \geq 0$.

Proof. If $0<\delta<1, x(0) \geq 0$, it follows from (3) that

$$
x(k) \geq 0
$$

for all $k \geq 0$. The proof is completed.

Lemma 2. Let $f_{1}(y)=(w y+h)^{2} /\left(s+v N y^{2}\right)$. It holds that $f_{1}(y)$ is increasing for all $y \in[0, w s / v N h]$.

Proof. Since

$$
\frac{d f_{1}}{d y}=\frac{2(w y+h)(w s-v N h y)}{\left(s+v N y^{2}\right)^{2}}
$$

we have $d f_{1} / d y \geq 0$ for all $y \in[0, w s / v N h]$.

\section{Invariant Set}

Definition 3. A compact set $S$ is called an invariant set of (3), if for any $C(0) \in S$, the trajectory of (3) starting from $C(0)$ will remain in $S$ for all $k \geq 0$.

An invariant set provides a method to guarantee nondivergence of trajectories. Denote that

$$
M=\max \left\{x(0), \max _{0 \leq y \leq \gamma}\left\{(1-\delta) y+\delta \frac{(w y+h)^{2}}{s+v N y^{2}}\right\}\right\} .
$$

Clearly, $M$ is a bounded constant.

Theorem 4. Suppose that $0<\delta<1$. If $x(0) \geq 0$, then $\Omega=$ $\{x \in R, 0 \leq x \leq M\}$ is an invariant set of (3).

Proof. Let $\gamma$ be the maximal real root of the following equation:

$$
v N y^{3}-w^{2} y^{2}+(s-2 w h) y-h^{2}=0
$$

Since $v N>0$, it follows from the Vieta theorem that $\gamma>0$.
In the following, by mathematical induction, we will prove that $x(k) \leq M$ for all $k \geq 0$; that is, $\Omega=\{x \in R, 0 \leq$ $x \leq M\}$ is an invariant set of (3).

Suppose that $0 \leq x(k) \leq M$; two cases are considered.

Case 1. If $x(k)>\gamma>0$, from $0<\delta<1$, we have

$$
0<1-\delta+\delta \frac{(w x(k)+h)^{2}}{s x(k)+v N x^{3}(k)}<1 .
$$

It follows from (3)-(8) that

$$
\begin{aligned}
x(k+1) & =(1-\delta) x(k)+\delta \frac{(w x(k)+h)^{2}}{s+v N x^{2}(k)} \\
& =\left[1-\delta+\delta \frac{(w x(k)+h)^{2}}{s x(k)+v N x^{3}(k)}\right] x(k) \\
& <x(k) \leq M .
\end{aligned}
$$

Case 2. If $0 \leq x(k) \leq \gamma$, it follows from (3) that

$$
x(k+1) \leq \max _{0 \leq x(k) \leq \gamma}\left\{(1-\delta) x(k)+\delta \frac{(w x(k)+h)^{2}}{s+v N x^{2}(k)}\right\} \leq M .
$$

From the forementioned, we have $0 \leq x(k+1) \leq M$ if $0 \leq x(k) \leq M$. It follows from mathematical induction that $0 \leq x(k) \leq M$ for all $k \geq 0$ if $0 \leq x(0) \leq M$. That is, $\Omega=\{x \in R, 0 \leq x \leq M\}$ is an invariant set of (3). This proof is completed.

Theorem 4 shows that the trajectory of (3) starting from any nonnegative value will always be bounded if $0<\delta<1$.

\section{Stability and Convergence Analysis}

In this section, we will study the stability and convergence of the network (3).

Definition 5. A point $x^{*} \in R$ is called a fixed point of (3), if and only if

$$
x^{*}=\frac{\left(w x^{*}+h\right)^{2}}{s+v N x^{* 2}} \text {. }
$$

Clearly, there may exist three fixed points. Thus, the study of dynamics belongs to a multistability problem [11].

According to the Lyapunov indirect method [12], a fixed point of a discrete-time nonlinear system is stable if the absolute of each eigenvalue of the Jacobian matrix of the system at this point is less than 1 . For the network (3), the eigenvalues of Jacobian matrix at each fixed point will be computed.

Theorem 6. Let $x^{*}$ be a fixed point of the network (3). If

$$
\begin{gathered}
w^{2} x^{* 2}+3 h^{2}>(2 s-4 w h) x^{*}, \\
\frac{2-\delta}{\delta} w^{2} x^{* 2}+\frac{2-3 \delta}{\delta} h^{2}>\left(\frac{4 \delta-4}{\delta} w h-2 s\right) x^{*},
\end{gathered}
$$

and both hold, then $x^{*}$ is stable. 
Proof. Let

$$
f(x)=(1-\delta) x+\delta \frac{(w x+h)^{2}}{s+v N x^{2}}
$$

We have

$$
\frac{d f}{d x}=1-\delta+\delta \frac{2 w(w x+h)\left(s+v N x^{2}\right)-2 v N x(w x+h)^{2}}{\left(s+v N x^{2}\right)^{2}} \text {. }
$$

It follows from (11) that

$$
\begin{aligned}
\left.\frac{d f}{d x}\right|_{x^{*}} & =1-\delta+\delta \frac{2 w h x^{*}+2 w^{2} x^{* 2}-2 v N x^{* 3}}{\left(w x^{*}+h\right)^{2}} \\
& =1-\delta+\delta \frac{(2 s-2 w h) x^{*}-2 h^{2}}{\left(w x^{*}+h\right)^{2}} .
\end{aligned}
$$

If inequality (12) holds, we have $\left.|d f| d x\right|_{x^{*}} \mid<1$. Thus, the fixed point $x^{*}$ is stable. The proof is completed.

Theorem 6 gives the stability conditions for a fixed point of (3). Specially, when the learning rate $0<\delta<1$, we will further study the convergence of the trajectories of (3).

Theorem 7. Suppose that $0<\delta<1$. If $\gamma \leq w s / v N h$ and $0 \leq x(0) \leq \gamma$, then the trajectories of (3) starting from $x(0)$ will converge to a fixed point, where $\gamma$ is the maximal real root of the following cubic equation:

$$
v N y^{3}-w^{2} y^{2}+(s-2 w h) y-h^{2}=0 .
$$

Proof. It follows from Definition 5 that a fixed point of (3) is the root of (16). Since $v N>0$, according to the Vieta theorem, there must exist a positive real root. In the following, we will prove the convergence of the trajectories of (3) in all three different cases, where $\Delta$ represents the discriminant of (16). For convenience, the maximal real root of (16) is denoted by $\gamma$ uniformly in all the cases. Clearly, $\gamma>0$.

Case 1. Suppose that $\Delta>0$. Clearly, there exist one real root and two imaginary roots; that is, $\gamma$ is the unique fixed point of (3).

Firstly, we will prove that $\Omega_{1}=\{x \in R, 0 \leq x \leq \gamma\}$ is an invariant set of (3). Suppose that $0 \leq x(k) \leq \gamma, 0<\delta<1$. It follows that

$$
v N x(k)^{3}-w^{2} x^{2}(k)+(s-2 w h) x(k)-h^{2} \leq 0 .
$$

That is,

$$
\frac{(w x(k)+h)^{2}}{s+v N x^{2}(k)} \geq x(k)
$$

By Lemma 1 and (3) and (18), it holds that

$$
\begin{aligned}
x(k+1) & \leq(1-\delta) \frac{(w x(k)+h)^{2}}{s+v N x^{2}(k)}+\delta \frac{(w x(k)+h)^{2}}{s+v N x^{2}(k)} \\
& \leq \max _{0 \leq x(k) \leq \gamma} \frac{(w x(k)+h)^{2}}{s+v N x^{2}(k)} .
\end{aligned}
$$

Since $\gamma \leq w s / v N h$, it follows from Lemma 2 that

$$
\max _{0 \leq x(k) \leq \gamma} \frac{(w x(k)+h)^{2}}{s+v N x^{2}(k)}=\frac{(w \gamma+h)^{2}}{s+v N \gamma^{2}}=\gamma
$$

From Lemma 1 and (19) and (20), we have $0 \leq x(k+1) \leq \gamma$. It follows from mathematical induction that $0 \leq x(k) \leq \gamma$ for all $k \geq 0$ if $0 \leq x(0) \leq \gamma$. That is, $\Omega_{1}=\{x \in R, 0 \leq x \leq \gamma\}$ is an invariant set of (3).

On the other hand, it follows from Lemma 1 and (3) and (18) that

$$
x(k+1) \geq(1-\delta) x(k)+\delta x(k)=x(k)
$$

if $0 \leq x(k) \leq \gamma$.

By the invariance of $\Omega_{1}$, it follows that inequality (21) holds for all $k \geq 0$ if $x(0) \in \Omega_{1}$. This means the sequence $\{x(k)\}$ is monotone increasing and bounded; thus, $\lim _{k \rightarrow+\infty} x(k)=\gamma$ if $0 \leq x(0) \leq \gamma$.

Case 2. Suppose that $\Delta=0$. There exist three real roots, two of them are equal. As analyzed in Case 1, it holds that $\lim _{k \rightarrow+\infty} x(k)=\gamma$ if $0 \leq x(0) \leq \gamma$.

Case 3. Suppose that $\Delta<0$. There exist three different real roots, and we denote them by $\gamma_{1}<\gamma_{2}<\gamma$. According to the Vieta theorem, the relationship of the three roots is either $\gamma_{1}<\gamma_{2} \leq 0<\gamma$ or $0 \leq \gamma_{1}<\gamma_{2}<\gamma$.

(1) Suppose that $\gamma_{1}<\gamma_{2} \leq 0<\gamma$, as analyzed in Case 1, and it holds that $\lim _{k \rightarrow+\infty} x(k)=\gamma$ if $0 \leq x(0) \leq \gamma$.

(2) Suppose that $0 \leq \gamma_{1}<\gamma_{2}<\gamma$. The initial conditions can be divided into the following three cases.

(a) If $0 \leq x(0) \leq \gamma_{1}$, similar to the analysis in Case 1 , we can obtain that $\Omega_{2}=\left\{x \in R, 0 \leq x \leq \gamma_{1}\right\}$ is an invariant set of (3). Since $x(k+1) \geq x(k)$ for all $k \geq 0$, it holds that $\lim _{k \rightarrow+\infty} x(k)=\gamma_{1}$.

(b) If $\gamma_{1} \leq x(0) \leq \gamma_{2}$, we will prove that $\lim _{k \rightarrow+\infty} x(k)=\gamma_{1}$. Firstly, we will show that $\Omega_{3}=\left\{x \in R, \gamma_{1} \leq x \leq \gamma_{2}\right\}$ is an invariant set of (3). Suppose that $\gamma_{1} \leq x(k) \leq \gamma_{2}, 0<\delta<1$. It follows that

$$
\frac{(w x(k)+h)^{2}}{s+v N x^{2}(k)} \leq x(k) .
$$

Since $\gamma_{1}<\gamma_{2}<\gamma \leq w s / v N h$, it holds from Lemmas 1-2, and (3) and (22) that

$$
\begin{aligned}
x(k+1) & \geq(1-\delta) \frac{(w x(k)+h)^{2}}{s+v N x^{2}(k)}+\delta \frac{(w x(k)+h)^{2}}{s+v N x^{2}(k)} \\
& \geq \min _{\gamma_{1} \leq x(k) \leq \gamma_{2}} \frac{(w x(k)+h)^{2}}{s+v N x^{2}(k)} \\
& =\frac{\left(w \gamma_{1}+h\right)^{2}}{s+v N \gamma_{1}^{2}}=\gamma_{1} .
\end{aligned}
$$


By (3) and (22), it holds that

$$
x(k+1) \leq(1-\delta) x(k)+\delta x(k)=x(k) \leq \gamma_{2}
$$

if $\gamma_{1} \leq x(k) \leq \gamma_{2}$. It follows from (23), (24), and mathematical induction that $\Omega_{3}$ is an invariant set of (3). From the invariant set $\Omega_{3}$ and (24), it can be concluded that $\lim _{k \rightarrow \infty} x(k)=\gamma_{1}$.

(c) If $\gamma_{2} \leq x(0) \leq \gamma$, we will show that $\lim _{k \rightarrow+\infty} x(k)=\gamma$. Firstly, $\Omega_{4}=\left\{x \in R, \gamma_{2} \leq\right.$ $x \leq \gamma\}$ will be shown to be an invariant set of (3). Suppose that $\gamma_{2} \leq x(k) \leq \gamma, 0<\delta<1$. Similar to the analysis in Case 1, it follows that

$$
x(k+1) \leq \max _{\gamma_{2} \leq x(k) \leq \gamma} \frac{(w x(k)+h)^{2}}{s+v N x^{2}(k)}=\gamma .
$$

From (3) and (18), we have

$$
x(k+1) \geq(1-\delta) x(k)+\delta x(k)=x(k) \geq \gamma_{2}
$$

if $\gamma_{2} \leq x(k) \leq \gamma$. By (25) and (26), it holds that $\Omega_{4}$ is an invariant set of (3). The invariant set $\Omega_{4}$ and (26) show that $\lim _{k \rightarrow+\infty} x(k)=\gamma$.

In summary, the trajectories of (3) will converge to a fixed point if $\gamma \leq w s / v N h$ and $0 \leq x(0) \leq \gamma$. The proof is completed.

Theorem 7 shows that the trajectories starting from $[0, \gamma]$ will converge. What will happen if $x(0)>\gamma$ ? We have the following theorem.

Theorem 8. Suppose that $0<\delta<1$. If $\gamma \leq w s / v N h$ and $x(0) \geq 0$, then the trajectories of (3) starting from $x(0)$ will converge to a fixed point, where $\gamma$ is the maximal real root of (16).

Proof. If $0 \leq x(0)>\gamma$, it follows from Theorem 7 that the trajectory starting from $x(0)$ will converge.

If $x(0) \geq \gamma>0$, it holds from (9) that there must exist an integer $K \geq 0$ such that $0 \leq x(K) \leq \gamma$. By Theorem 7 , it follows that the trajectory starting from $x(K)$ will converge to a fixed point of (3). The proof is completed.

Theorem 8 gives the conditions guaranteeing the convergence of (3). If $0<\delta<1$ and $\gamma \leq w s / v N h$, then the trajectory starting from any nonnegative real number will converge to a fixed point of (3).

Next, we will give an example to further illustrate the previous analysis.

Example 9. Consider the network (3) with $s=41, w=1.15$, $v N=0.0128$, and $\delta=0.3$.

Condition (a) (where $h=8$ ). It follows from (16) that $\Delta<0$. It is easy to check that there are three fixed points of the network (3). By Theorem 6, it holds that $\gamma_{1}=3.5401$ and $\gamma=82.7021$ are stable while $\gamma_{2}=17.0781$ is unstable. The unstable fixed point acts as a threshold that separates two stable fixed points. Since $\gamma<w s / v N h=454.7647$, it follows from Theorem 7 that the trajectories starting from $\left[0, \gamma_{2}\right)$ should converge to $\gamma_{1}=3.5401$, while the trajectories starting from $\left[\gamma_{2}, \gamma\right]$ should converge to $\gamma=82.7021$. Figure 1 illustrates these results.

Condition $(b)$ (where $h=13$ ). In this condition, $\Delta>$ $0, \gamma=95.7004<w s / v N h=283.3534$. It follows from Theorem 7 that the trajectories starting from any nonnegative value should converge to the fixed point $\gamma=95.7004$. Figure 2 illustrates the result.

The only difference between the previous two conditions is the background input $h$. Figures 1 and 2 show that the low-rate disappears with an increase in background inputs, switching the network between regimes with one or two stable uniform rates.

\section{Bifurcation and Chaos}

With the increase in background input $h$, the network (3) may exhibit more complicated dynamical properties. To illustrate this, we will draw the bifurcation diagram of the network (3) and calculate Lyapunov exponents of the network.

Example 10. Consider the network (3) with $s=8, w=1.2$, and $v N=40$.

Condition (a) (where $\delta=0.9$ ). Theorem 4 guarantees the nondivergence of the network (3). Since $\Delta>0$, then there exists only one fixed point. With the increase of the background input $h$, the changes of the dynamical behavior of network (3) are illustrated in Figure 3. It can be seen that $h \simeq 4.7$ is a bifurcation point of network (3), where the fixed point becomes unstable and a 2 -periodic orbit shows up. As $h \simeq 176$, the 2 -periodic orbit becomes unstable and a 4 period orbit appears. The 4-period orbits are always stable until $h=600$.

Condition $(b)$ (where $\delta=0.99$ ). The bifurcation diagram of network (3) is shown in Figure 4 . To further show the route to chaos with the increase of $h$, another bifurcation diagram is given in Figure 5. The only difference in Figures 4 and 5 is the value range of $h$. Figure 4 shows that $h \simeq 4.2$ is a bifurcation point. From Figure 5, we can see that $h \simeq 36$ is also a bifurcation point, where the 2-period orbit becomes unstable and a 3-period orbit shows up, and then 5-period orbit appears, and so forth; as $h$ approaches 103, the network enters chaotic state.

To further illustrate the chaotic property, the Lyapunov exponents are computed according to Dror and Tsodyks [13]. For each value of $h$, the Lyapunov exponent of the network (3) is plotted in Figure 6. As $h \simeq 103$, the Lyapunov exponent is positive and the network becomes chaotic.

From Examples 9 and 10, we can see that with the change of background input, the discrete-time background neural network with uniform firing rate may switch among several dynamical states: single fixed point, periodic orbits, and chaos.

\section{Conclusions}

The discrete-time background network obtained by Euler method is investigated in this paper. The nondivergence, 


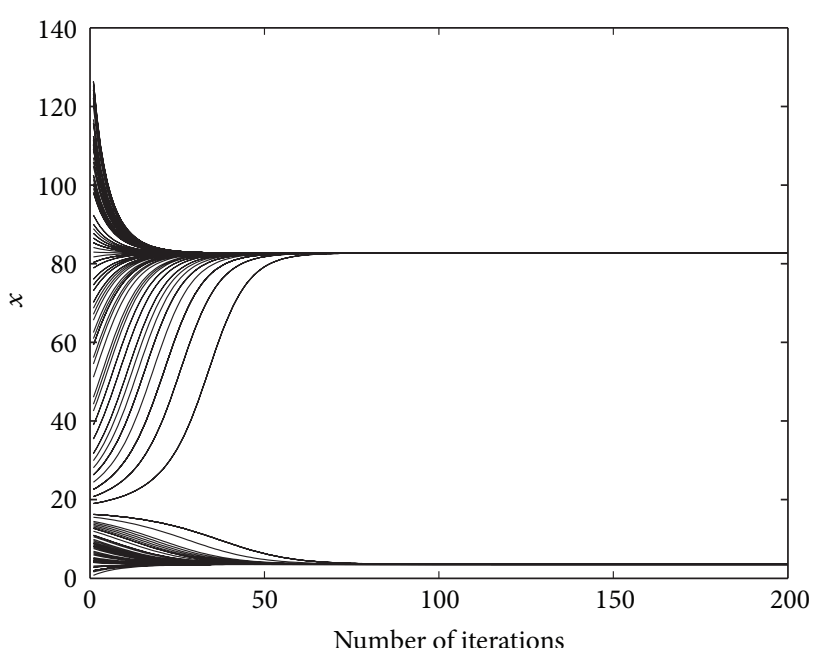

FIGURE 1: The convergence of the network (3) with $\delta=0.3, s=41$, $w=1.15$, and $v N=0.0128$, where $h=8$.

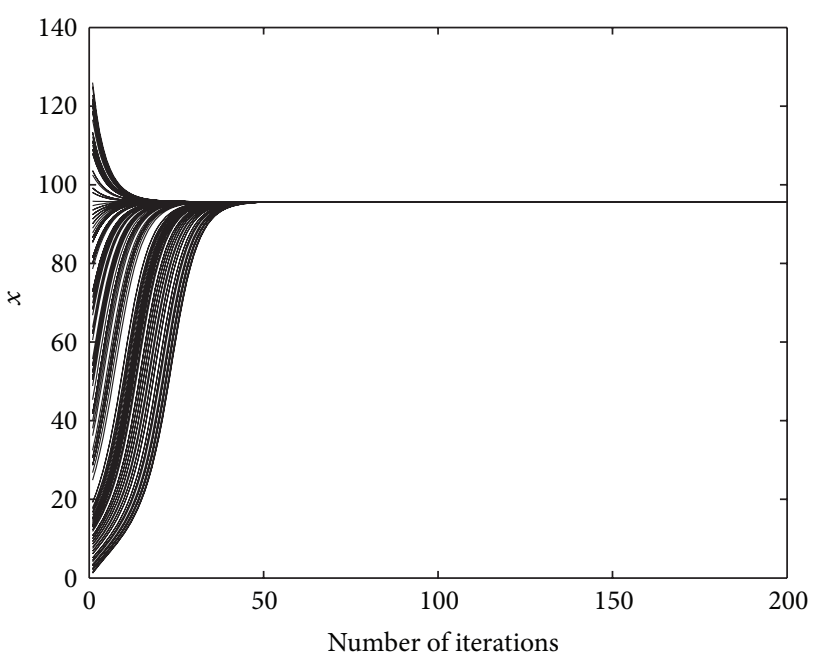

FIGURE 2: The convergence of the network (3) with $\delta=0.3, s=$ $41, w=1.15$, and $v N=0.0128$, where $h=13$.

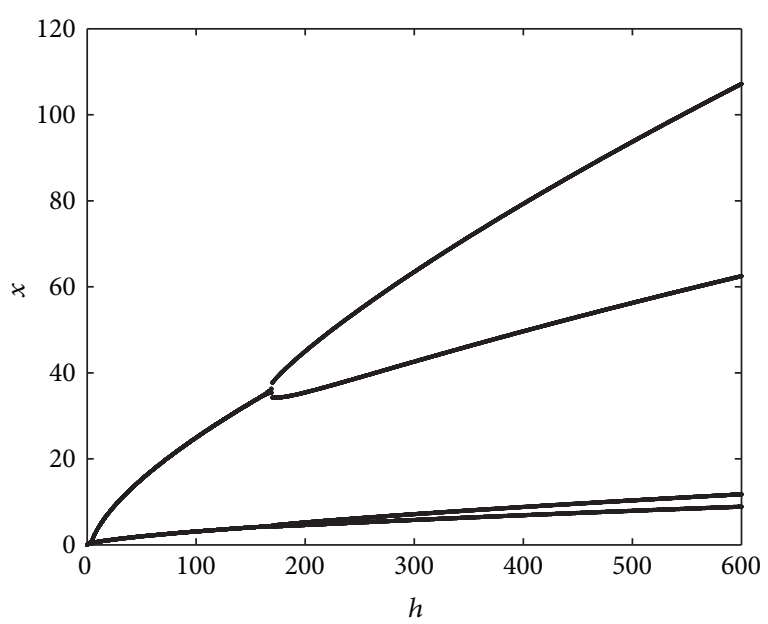

FIGURE 3: Bifurcation diagram of (3) with $s=8, w=1.2, v N=40$, and $\delta=0.9$.

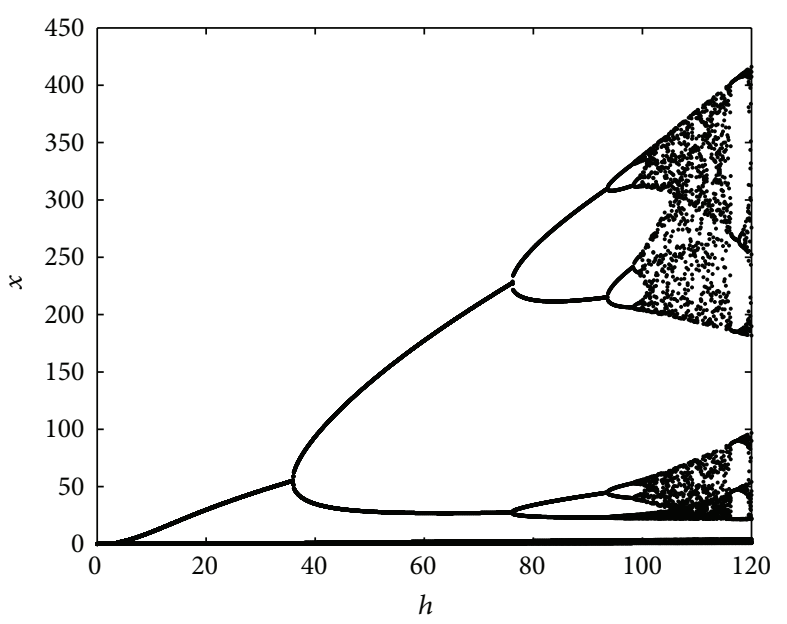

Figure 4: Bifurcation diagram of (3) with $s=8, w=1.2, v N=40$, and $\delta=0.99$.

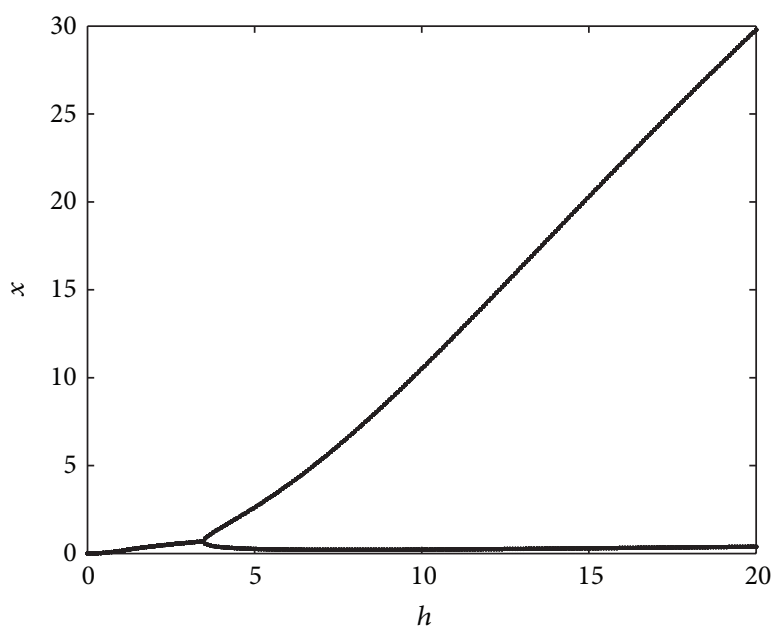

FIgURE 5: Bifurcation diagram of (3) with $s=8, w=1.2 v N=40$, and $\delta=0.99$

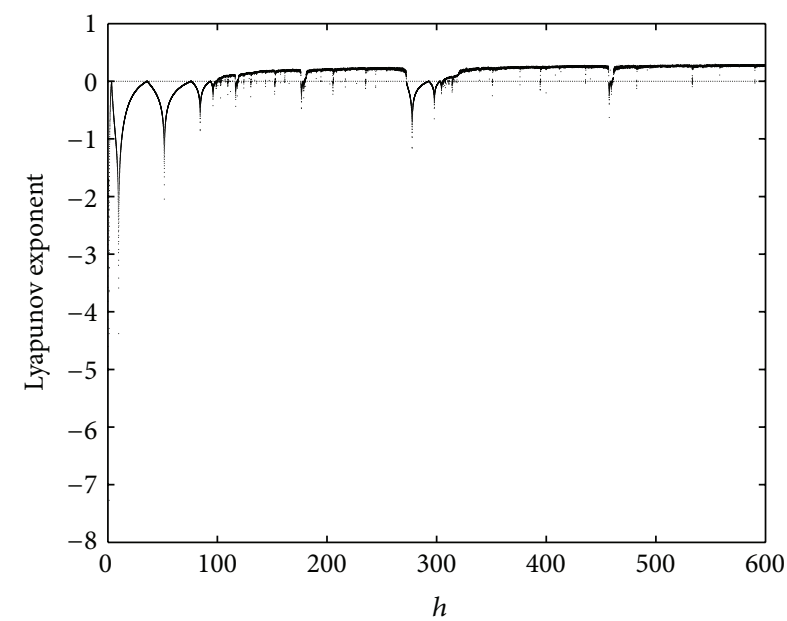

FIGURE 6: Lyapunov exponents of the network (3) with $s=8, w=$ $1.2, v N=40$, and $\delta=0.99$. 
stability, and convergence of the network are studied, respectively. As shown in this paper, though the nonlinear system is one dimensional, it is not easy to study its convergence. Furthermore, as the background input $h$ varies, it is shown that the network may exhibit complex dynamical behaviors such as bifurcation and chaos. This means that the dynamics of the discrete-time network can produce a much richer set of patterns than those discovered in continuous-time network. Since the background networks originate from the study of the activities of brain and chaotic activities are ubiquitous in the human brain, the chaos analysis of the background networks is significant.

\section{Acknowledgments}

This work was supported by the National Science Foundation of China (Grant nos. 61103168 and 61202045), the Scientific Research Foundation of Sichuan Provincial Department of education (Grant nos. 12ZB134), the Key Scientific Research Foundation of Xihua University (Grant no. Z1122633), and the Key Scientific Research Foundation of Sichuan Provincial Department of Education (Grant no. 11ZA004).

\section{References}

[1] E. Salinas, "Background synaptic activity as a switch between dynamical states in a network," Neural Computation, vol. 15, no. 7, pp. 1439-1475, 2003.

[2] D. Liu, S. Hu, and J. Wang, "Global output convergence of a class of continuous-time recurrent neural networks with timevarying thresholds," IEEE Transactions on Circuits and Systems II, vol. 51, no. 4, pp. 161-167, 2004.

[3] X. B. Liang and J. Si, "Global exponential stability of neural networks with globally Lipschitz continuous activations and its application to linear variational inequality problem," IEEE Transactions on Neural Networks, vol. 12, no. 2, pp. 349-359, 2001.

[4] H. Lu and G. Chen, "Global exponential convergence of multitime-scale neural networks," IEEE Transactions on Circuits and Systems II, vol. 52, no. 11, pp. 761-765, 2005.

[5] Z. Yi, P. A. Heng, and A. W. C. Fu, "Estimate of exponential convergence rate and exponential stability for neural networks," IEEE Transactions on Neural Networks, vol. 10, no. 6, pp. 14871493, 1999.

[6] L. Zhang and Z. Yi, "Dynamical properties of background neural networks with uniform firing rate and background input," Chaos, Solitons and Fractals, vol. 33, no. 3, pp. 979-985, 2007.

[7] M. G. Kitzbichler, M. L. Smith, S. R. Christensen, and E. Bullmore, "Broadband criticality of human brain network synchronization,” PLoS Computational Biology, vol. 5, no. 3, Article ID e1000314, 2009.

[8] D. R. Chialvo, "Emergent complex neural dynamics," Nature Physics, vol. 6, no. 10, pp. 744-750, 2010.

[9] K. Schwab, T. Groh, M. Schwab, and H. Witte, "Nonlinear analysis and modeling of cortical activation and deactivation patterns in the immature fetal electrocorticogram," Chaos, vol. 19, no. 1, Article ID 015111, 2009.

[10] O. Sporns, D. R. Chialvo, M. Kaiser, and C. C. Hilgetag, "Organization, development and function of complex brain networks," Trends in Cognitive Sciences, vol. 8, no. 9, pp. 418425, 2004.

[11] Z. Yi and K. K. Tan, Convergence Analysis of Recurrent Neural Networks, vol. 13 of Network Theory and Applications, Kluwer Academic, Boston, Mass, USA, 2004.

[12] H. N. Agiza, "On the analysis of stability, bifurcation, chaos and chaos control of Kopel map," Chaos, Solitons and Fractals, vol. 10, no. 11, pp. 1909-1916, 1999.

[13] G. Dror and M. Tsodyks, "Chaos in neural networks with dynamic synapses,” Neurocomputing, vol. 32-33, pp. 365-370, 2000. 


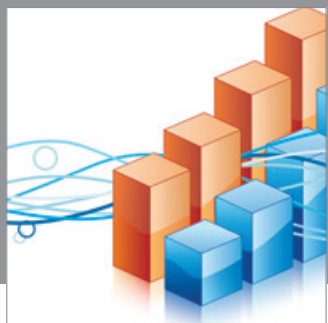

Advances in

Operations Research

mansans

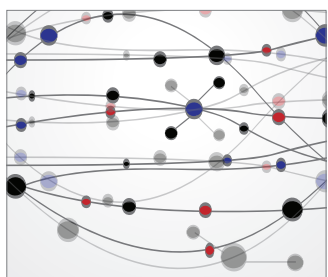

The Scientific World Journal
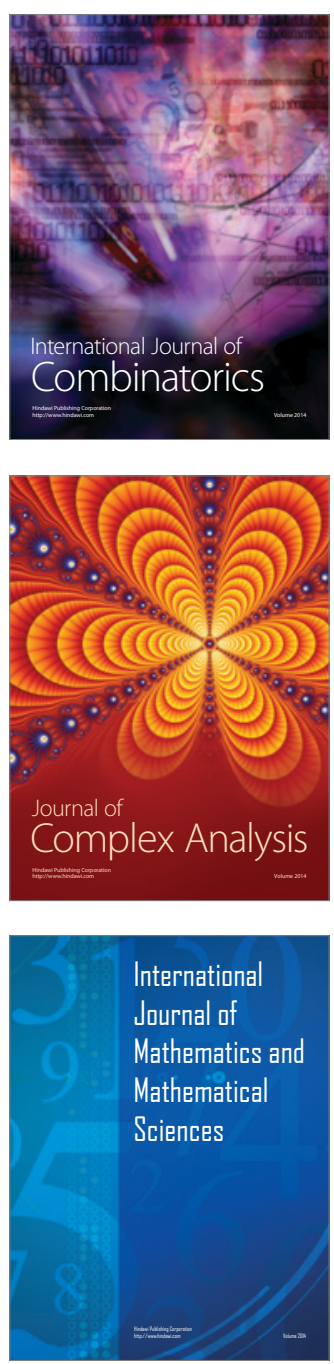
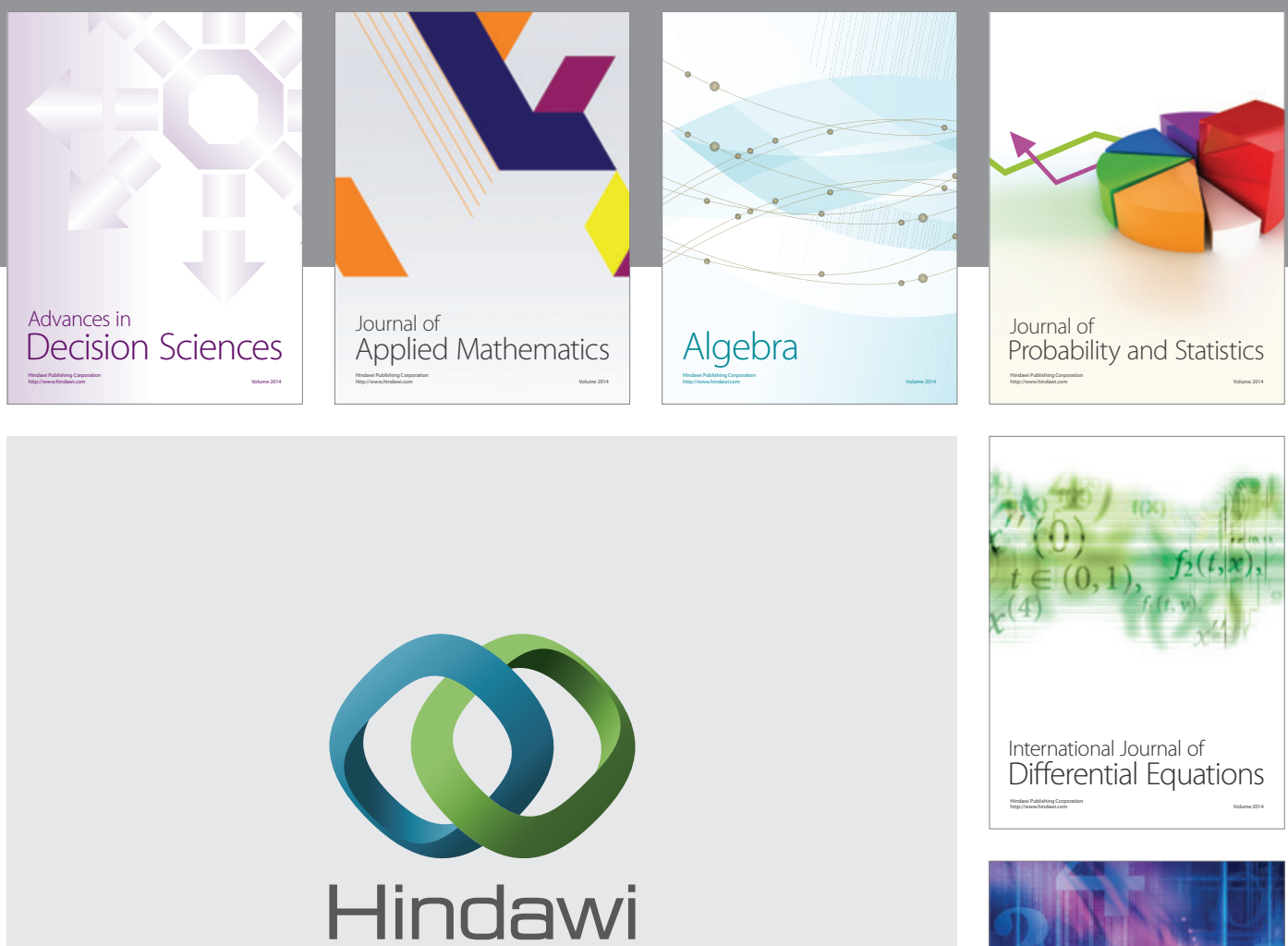

Submit your manuscripts at http://www.hindawi.com
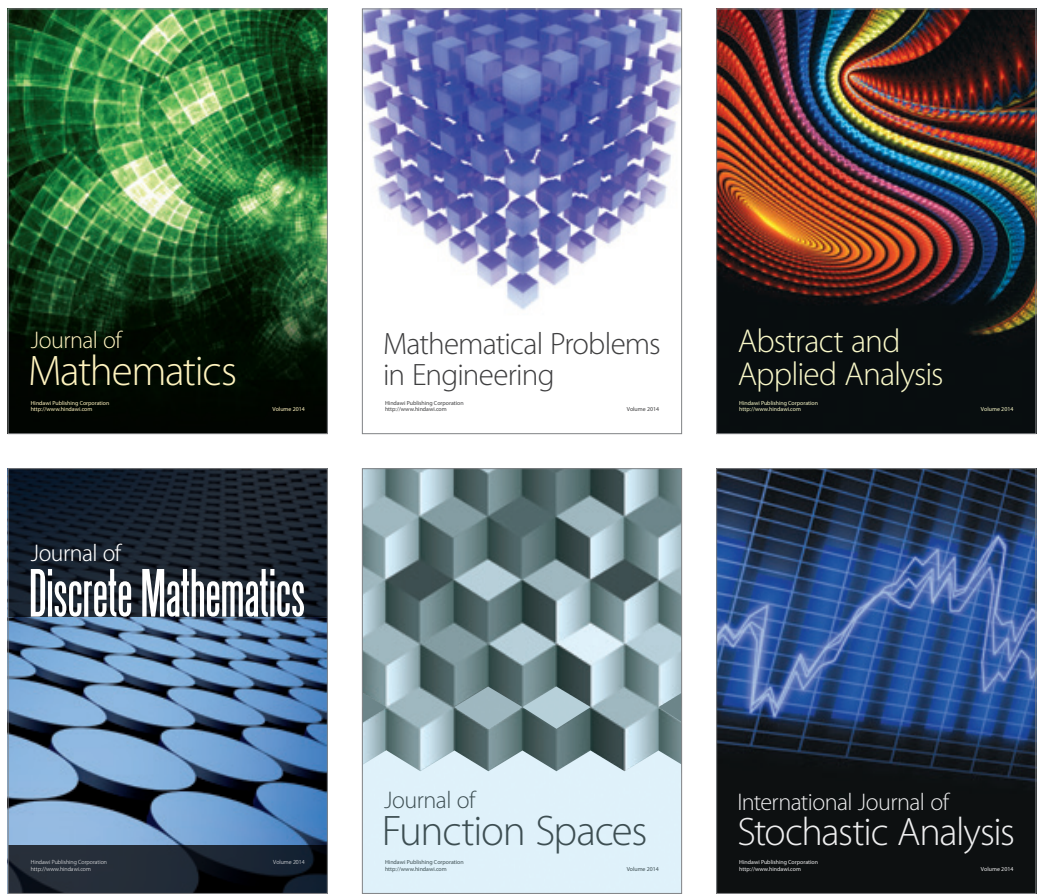

Journal of

Function Spaces

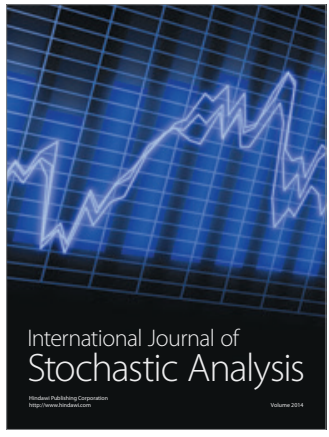

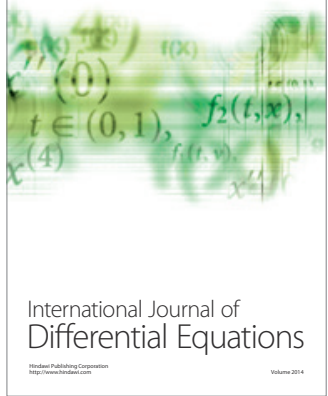
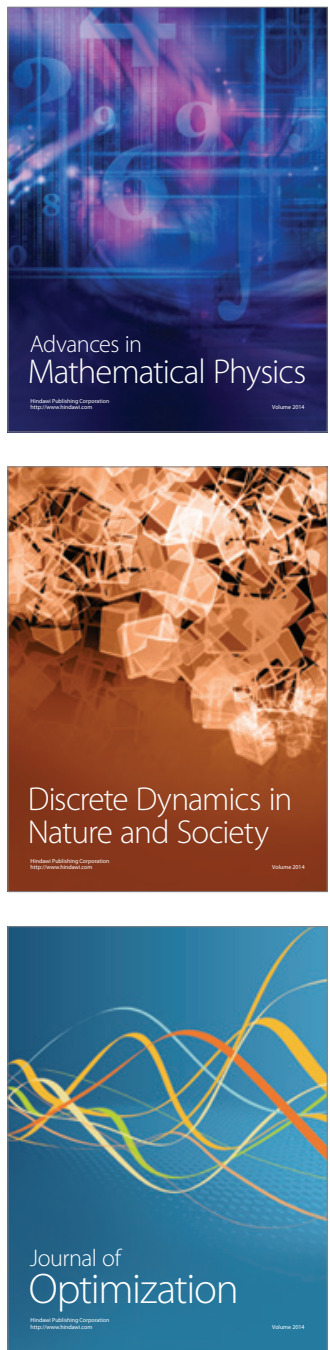